\title{
TissueCypher Barrett's esophagus assay impacts clinical decisions in the management of patients with Barrett's esophagus
}

\section{(ㄷ)(1) $(9$}

\author{
Authors \\ Institutions \\ 1 Department of Gastroenterology, Geisinger Health \\ System, Danville, Pennsylvania, United States \\ 2 Cernostics, Inc., Pittsburgh, Pennsylvania, United States
}

David L. Diehl' ${ }^{1}$, Harshit S. Khara ${ }^{1}$, Nasir Akhtar ${ }^{1}$, Rebecca J. Critchley-Thorne ${ }^{2}$

submitted 30.7 .2020

accepted after revision 21.10 .2020

Bibliography

Endoscopy International Open 2021; 09: E348-E355

DOI 10.1055/a-1326-1533

ISSN 2364-3722

(C) 2021. The Author(s).

This is an open access article published by Thieme under the terms of the Creative Commons Attribution-NonDerivative-NonCommercial License, permitting copying and reproduction so long as the original work is given appropriate credit. Contents may not be used for commecial purposes, or adapted, remixed, transformed or built upon. (https://creativecommons.org/licenses/by-nc-nd/4.0/)

Georg Thieme Verlag KG, Rüdigerstraße 14,

70469 Stuttgart, Germany

Corresponding author

David L. Diehl, MD, Department of Gastroenterology, 100 N. Academy Avenue, Geisinger Health System, Danville, PA, USA Fax: +1-412-774-7319

dldiehl@geisinger.edu

\section{ABSTRACT}

Background and study aims The TissueCypher Barrett's Esophagus Assay is a novel tissue biomarker test, and has been validated to predict progression to high-grade dyspla- sia (HGD) and esophageal adenocarcinoma (EAC) in patients with Barrett's esophagus (BE). The aim of this study was to evaluate the impact of TissueCypher on clinical decision-making in the management of BE.

Patients and methods TissueCypher was ordered for 60 patients with non-dysplastic (ND, $n=18$ ) $B E$, indefinite for dysplasia (IND, $n=25$ ), and low-grade dysplasia (LGD, $n=$ 17). TissueCypher reports a risk class (low, intermediate or high) for progression to HGD or EAC within 5 years. The impact of the test results on $\mathrm{BE}$ management decisions was assessed.

Results Fifty-two of 60 patients were male, mean age 65.2 \pm 11.8 , and 43 of 60 had long segment BE. TissueCypher results impacted $55.0 \%$ of management decisions. In $21.7 \%$ of patients, the test upstaged the management approach, resulting in endoscopic eradication therapy (EET) or shorter surveillance interval. The test downstaged the management approach in $33.4 \%$ of patients, leading to surveillance rather than EET. In the subset of patients whose management plan was changed, upstaging was associated with a high-risk TissueCypher result, and downstaging was associated with a low-risk result $(P<0.0001)$.

Conclusions TissueCypher was used as an adjunct to support a surveillance-only approach in $33.4 \%$ of patients. Upstaging occurred in $21.7 \%$ of patients, leading to therapeutic intervention or increased surveillance. These results indicate that the TissueCypher test may enable physicians to target EET for TissueCypher high-risk BE patients, while reducing unnecessary procedures in TissueCypher low-risk patients.

\section{Introduction}

Barrett's esophagus (BE) is a known precursor of esophageal adenocarcinoma (EAC), which has a rapidly rising incidence and a 5-year survival rate of less than $20 \%$ [1,2]. Endoscopic surveillance with biopsies and expert histologic review is recommended by gastrointestinal societies to identify dysplasia and early EAC when endoscopic eradication therapy (EET) can effectively prevent progression [3-5]. The effectiveness of this approach is limited by the random nature of endoscopic biopsy sampling, which may miss dysplastic areas, and by significant interobserver variation in histologic evaluation of biopsies [6, 7]. A diagnosis of low-grade dysplasia (LGD) confirmed by a gastrointestinal subspecialist pathologist is a significant predictor of progression to high-grade dysplasia (HGD) and EAC, and current society guidelines recommend EET or increased surveillance for patients with BE who have confirmed LGD [4, 8]. However, the reported progression rates for confirmed LGD vary widely and overdiagnosis of LGD is common [6,7,9-11], making it unclear whether therapeutic intervention is warranted 
for all patients with LGD. Pathologic analysis also can be confounded by cases that are difficult to diagnose, for example, if there is a background of inflammation, which can result in a diagnosis of "indefinite" for dysplasia (IND). These IND cases also present a management challenge because there is substantial heterogeneity between studies reporting on the progression risk associated with IND [12].

The rate of malignant progression from non-dysplastic (ND) $\mathrm{BE}$ is very low $[13,14]$, and clinical factors such as segment length, sex, and age may help guide decisions regarding surveillance intervals [15-17]. However, a subset of patients with ND BE will progress to HGD/EAC during the recommended 3-to 5-year surveillance interval, resulting in significant uncertainty and anxiety regarding management, as well as over-utilization of endoscopic surveillance that has not been effective in reducing the incidence and mortality of EAC $[18,19]$. There is a need for objective tests to identify BE patients with ND, IND or LGD who are at increased risk for progression. These patients could benefit from early EET to prevent progression, or increased surveillance to detect dysplasia earlier. Furthermore, a test that could identify patients who are at very low risk for progression could prevent unnecessary EET and over-surveillance.

The TissueCypher Barrett's Esophagus Assay has been developed and validated in five multi-institutional studies to predict progression from ND, IND, and LGD to HGD and EAC, and also to detect prevalence of $\mathrm{HGD} / \mathrm{EAC}$ in $\mathrm{BE}$ that can be missed on surveillance endoscopy [20-24]. TissueCypher utilizes a multiplexed fluorescence imaging platform that objectively extracts quantitative data on multiple epithelial, stromal, and morphometric features in intact tissue specimens $[25,26]$. TissueCypher integrates quantitative image analysis data and provides a risk score ranging from 0 to 10 that is used to classify patients as low-, intermediate- and high-risk for progression to HGD/ EAC within 5 years. This three-tier risk stratification approach may provide adjunctive evidence supporting EET in for BE patients with BE with high-risk TissueCypher scores, while also enabling a surveillance-only approach or extension of surveillance intervals in patients with low-risk scores. The predictive performance of TissueCypher in BE has been well described; however, the extent to which the test influences the management of patients with BE has not yet been studied. The objectives of this study were to determine whether TissueCypher results impact physician decision-making in the management of BE and to characterize these changes in terms of altering endoscopic surveillance intervals and use of EET.

\section{Patients and methods}

A prospective study was conducted that collected information on decisions made by two physicians regarding management of $\mathrm{BE}$ prior to and after receiving results from the TissueCypher Barrett's Esophagus Assay. TissueCypher is a commercially available test for physicians managing patients with $\mathrm{BE}$. The test is performed on slide blanks recut from existing tissue blocks of biopsies or mucosal resections for $B E$, which are sent to the Cernostics clinical laboratory (CLIA\#: 39D2110302, Pittsburgh, Pennsylvania, United States) for testing. TissueCypher integrates quantitative image analysis data from nuclear morphology and nine protein-based biomarkers ( $p 16$, alpha-methylacyl-coA racemase [AMACR], p53, CD68, cyclooxygenase [COX]-2, CD45RO, hypoxia inducible factor [HIF]-1alpha, HER2 / neu and cytokeratin-20 [K20]). A clinical report is generated that includes a risk score ranging from 0 to 10 , with 0 indicating lowest risk and 10 indicating highest risk. This score is then stratified into three risk classes (low 0 to $<5.5$, intermediate 5.5 to $<6.4$, or high 6.4 to 10 ) that predict progression to HGD/EAC within 5 years [20]. The results provided are adjunctive to the ordering physician's workup for patients with BE.

TissueCypher tests ordered at Geisinger Medical Center between June 2016 and April 2020 were considered for this study. A pre-TissueCypher management plan survey was sent to physicians after receipt of the test order. The survey included the following endoscopic surveillance interval options: 3 and 6 months and 1,3 , or 5 years, or other and the following treatment options: no treatment, mucosal resection, radiofrequency ablation, cryotherapy, esophagectomy, referral to oncologist, and other (free text describing the treatment plan). A post-TissueCypher management plan survey was then sent after the test results were reported.

Additional data collected were age at the time the test was ordered, sex, pathologic diagnosis of biopsies for which the test was ordered, worst historical pathologic diagnosis, Prague classification, segment length (short $<3 \mathrm{~cm}$, long $\geq 3 \mathrm{~cm}$ ), sex, and indication/reason for ordering the test. Indications for ordering the test could be grouped into categories of support for a donot-treat strategy in LGD, support for a do-not-treat strategy in other situations, risk stratification for IND, evaluation of persistent intestinal metaplasia after EET, and clinical/endoscopic concern. "Clinical/endoscopic concern" included patients with worrisome endoscopic features (such as polypoid lesions, or abnormal appearing but non-nodular mucosa), a history of intramucosal carcinoma, or very discordant pathological results from initial outside biopsy compared to repeat biopsy at referral center.

The pretest management plans (endoscopic surveillance interval and treatment plan) were compared to the management plans after receiving the test results to determine whether management decisions were changed by the test results, and to characterize the changes. Pretest and post-test management recommendations were recorded by two gastroenterologists (DLD and HSK) at a single center. Only patients with both pre- and post-TissueCypher management recommendations were included in the analysis. Management changes were characterized as upstaged if the plan changed from surveillanceonly to therapeutic intervention, additional therapy was recommended, ongoing therapy or surveillance was recommended where discontinuation was being considered, and/or increased surveillance frequency was recommended. Management changes were characterized as downstaged if the plan was changed from therapeutic intervention to a surveillance-only approach, or the surveillance frequency was reduced after review of test results. A chi-squared test was used to evaluate associations between categorical variables. The study protocol was reviewed and approved by the Geisinger Health System In- 
86 TissueCypher tests ordered

Excluded 5 patients with tests ordered for

$\rightarrow$ specimens with expert Dx higher than LGD and 4 for whom the test was not reportable

77 patients with tests ordered for specimens with expert Dx ND, IND or LGD

Excluded 17 patients lacking pre- and/or post-TissueCypher BE management decisions

60 patients with complete pre- and post-TissueCypher $\mathrm{BE}$ management decisions were analyzed

- Fig. 1 Flowchart of included/excluded patients.

stitutional Review Board (IRB), Danville, Pennsylvania, United States. All authors had access to the study data and reviewed and approved the final manuscript.

\section{Results}

\section{Patient characteristics}

Pretest and post-test management recommendations were obtained for 60 patients with BE and a diagnosis of ND $(n=18)$, IND $(n=25)$ or LGD $(n=17)$ diagnosed by a gastrointestinal subspecialist pathologist at Geisinger Medical Laboratories. The flowchart in $\mathbf{F i g .} 1$ shows the tests that were included or excluded from the analysis. The mean age of the included patients was $65.2 \pm 11.8$ (range 35.1 to 85.0 ), 52 of 60 patients $(86.7 \%)$ were male, and 43 of 60 patients $(71.7 \%$ ) had long segment Barrett's esophagus ( $\triangleright$ Table 1 ). There were no significant differences in age, sex, histologic diagnoses, or the ordering physician between the included patients and the patients who were excluded due to lack of pre-management and post-management data, indicating that the exclusions did not introduce bias. Indications for ordering TissueCypher were clinical/endoscopic concern $(n=13)$, support of a do-not-treat strategy in LGD $(n=11)$, support of a do-not-treat strategy in other situations $(n=8)$, risk stratification of IND $(n=25)$, and evaluation of persistent intestinal metaplasia after EET $(n=3$, patients had

\begin{tabular}{|c|c|}
\hline \multicolumn{2}{|l|}{ Age } \\
\hline Mean \pm SD & $65.2 \pm 11.8$ \\
\hline Range & $35.1-85$ \\
\hline \multicolumn{2}{|l|}{ Sex, n (\%) } \\
\hline - Male & $52(86.7)$ \\
\hline - Female & $8(13.4)$ \\
\hline \multicolumn{2}{|l|}{ Segment length, $\mathrm{n}(\%)$} \\
\hline - Short & $17(28.4)$ \\
\hline - Long & $43(71.7)$ \\
\hline \multicolumn{2}{|l|}{ Prague classification } \\
\hline - C, Mean (range) & $4.7(0,16)$ \\
\hline - M, Mean (range) & $6.3(1,16)$ \\
\hline \multicolumn{2}{|l|}{ Expert Dx, n (\%) } \\
\hline - ND & $18(30)$ \\
\hline - IND & $25(41.7)$ \\
\hline - LGD & $17(28.4)$ \\
\hline \multicolumn{2}{|c|}{ TissueCypher results, n (\%) } \\
\hline - Low-risk & $39(65)$ \\
\hline - Intermediate-risk & $7(11.7)$ \\
\hline - High-risk & $14(23.3)$ \\
\hline \multicolumn{2}{|c|}{$\begin{array}{l}\text { SD, standard deviation; Prague Classification, C: circumferential length anc } \\
\text { M: maximal length; Dx, pathologic diagnosis; ND, non-dysplastic; IND, in- } \\
\text { definite for dysplasia; LGD, low-grade dysplasia. }\end{array}$} \\
\hline
\end{tabular}

complete eradication of dysplasia but not complete eradication of intestinal metaplasia after at least one round of ablation). Repeat endoscopy with biopsies for patients with IND was not done. All patients with diagnosis of IND were on chronic proton pump inhibitor (PPI) treatment, and the TissueCypher test was ordered at the first diagnosis of IND or after repetitive diagnoses of IND. The TissueCypher test reported a result of low-risk for 39 patients (65\%), intermediate-risk for seven patients (11.7\%), and high-risk for 14 patients (23.3\%) (> Table 2 ).

To determine the change in physician behavior due to the test results, the post-test management recommendations

> Table 2 TissueCypher Results in Diagnostic Classes

\begin{tabular}{|l|l|l|l|}
\hline & All patients & ND patients & IND patients \\
\hline & $\mathbf{n}=\mathbf{6 0}$ & $\mathbf{n = 1 8}$ & $\mathbf{n}=\mathbf{2 5}$ \\
\hline Low-risk & $39(65)$ & $15(83.3)$ & $\mathbf{n}=\mathbf{1 7}$ \\
\hline Intermediate-risk & $7(11.7)$ & $2(11.1)$ & $4(16)$ \\
\hline High-risk & $14(23.3)$ & $1(5.6)$ & $4(16)$ \\
\hline BE, Barrett's esophagus; ND, non-dysplastic; IND, indefinite for dysplasia; LGD, low-grade dysplasia. & $9(52.9)$ \\
\hline
\end{tabular}




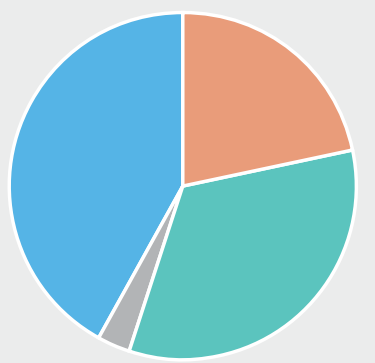

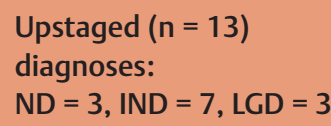

Downstaged $(n=20)$ diagnoses: $\mathrm{ND}=6, \mathrm{IND}=8, \mathrm{LGD}=6$

\section{Other $(\mathrm{n}=2)^{*}$}

diagnoses: $L G D$

No change $(n=25)$
diagnoses:
$N D=9$, IND $=10, L G D=6$

No change $(n=25)$

diagnoses:
- Fig. 2 Impact of TissueCypher results on management decisions. Upstaged management indicates that the test results led to increased surveillance frequency and/or a change from no treatment to recommendation of therapeutic intervention. Downstaged management indicates that the test results led to decreased surveillance frequency and/or a change from planned therapeutic intervention to recommendation of no treatment. * Treatment of newly diagnosed co-morbidity took priority over BE management.

were compared to the pretest management recommendations. TissueCypher results had a statistically significant impact on decision-making regarding management of BE. Following review of TissueCypher test results, the management plan chan-

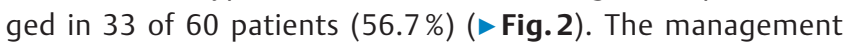
plan was upstaged in eight of 14 patients $(57.1 \%$ ) who had a high-risk TissueCypher score, and the management was downstaged in 19 of 39 patients $(48.7 \%)$ with a low-risk score $(P<$ $0.0001)$. Eighteen of 39 patients who scored low-risk were not downstaged due to other clinical/endoscopic concerns $(n=7)$, diagnosis of IND $(n=8)$ or because the test was ordered in support of a do-not-treat strategy $(n=3)$. In the two upstaged patients who scored low-risk, the recommended surveillance in- terval was reduced from 3 years to 1 year upon review of test results. In the subset of 33 patients whose management plan was changed after receipt of test results, upstaging was significantly associated with a high-risk test result, and downstaging was associated with a low-risk result $(P<0.0001)$.

The management plan was upstaged after review of test results in 13 patients (21.7\%) ( $\mathbf{F i g . 2}$ ), resulting in a variety of changes in management plan: 1 ) a change from surveillanceonly to therapeutic intervention with ablation therapy $(n=7)$; 2) recommendation for endoscopic mucosal resection (EMR) in addition to ablation $(n=1) ; 3)$ fundoplication and shorter surveillance interval (from 1 year to 6 months) $(n=1)$; 4) shorter surveillance interval (from 3 years to 1 year) $(n=2) 5$ ) ongoing ablation where a surveillance-only plan was being considered $(n=1)$; and 6$)$ ongoing surveillance versus discontinuation of surveillance $(n=1)$. In the group of patients whose management was upstaged, eight scored TissueCypher high-risk, three intermediate-risk, and two low-risk, indicating that the majority of patients were upstaged due to a high-risk test result. Three of the 13 upstaged patients had a diagnosis of LGD, seven were IND and three had ND BE.

The management plan was downstaged after test results were reviewed in 20 patients (33.4\%), resulting in a change from therapeutic intervention with ablation to recommendation of a surveillance-only plan in 19 patients, and extension of surveillance intervals (from 1 year to 3 years) in one patient ( $\vee$ Fig.2). In the subset of patients whose management was downstaged after review of the test results, 19 scored TissueCypher low-risk and one scored intermediate-risk, suggesting that the majority of downstaged management decisions were due to a low-risk test result. The management plan was not impacted for 25 patients $(42.4 \%)$ of whom 18 scored TissueCypher low-risk, three scored intermediate-risk, and four scored high-risk. Two patients were diagnosed with a comorbidity shortly after the test results were delivered, and treatment of the comorbidity took priority over management of BE.

The impact of the test results was not significantly different between the three diagnostic classes of ND, IND and LGD ( $P=$ 0.8904) ( $\triangleright$ Table 3 ). In patients with ND BE, $50 \%$ of management plan decisions were changed after review of test results

- Table 3 Impact of TissueCypher on BE management decisions: Change in management ${ }^{1}$

\begin{tabular}{|c|c|c|c|c|}
\hline & All patients & ND patients & IND patients & LGD patients \\
\hline & $n=60$ & $n=18$ & $n=25$ & $n=17$ \\
\hline Upstaged management & $13(21.7)$ & $3(16.7)$ & $7(28)$ & $3(17.6)$ \\
\hline Downstaged management & $20(33.4)$ & $6(33.3)$ & $8(32)$ & $6(35.3)$ \\
\hline No change & $25(41.7)$ & $9(50)$ & $10(40)$ & $6(35.3)$ \\
\hline Other & $2(3.4)$ & $0(0)$ & $0(0)$ & $2(11.8)$ \\
\hline Changed (up/down) & $33(55)$ & $9(50)$ & $15(60)$ & $9(52.9)$ \\
\hline Not changed (none/other) & $27(45)$ & $9(50)$ & $10(40)$ & $8(47.1)$ \\
\hline
\end{tabular}



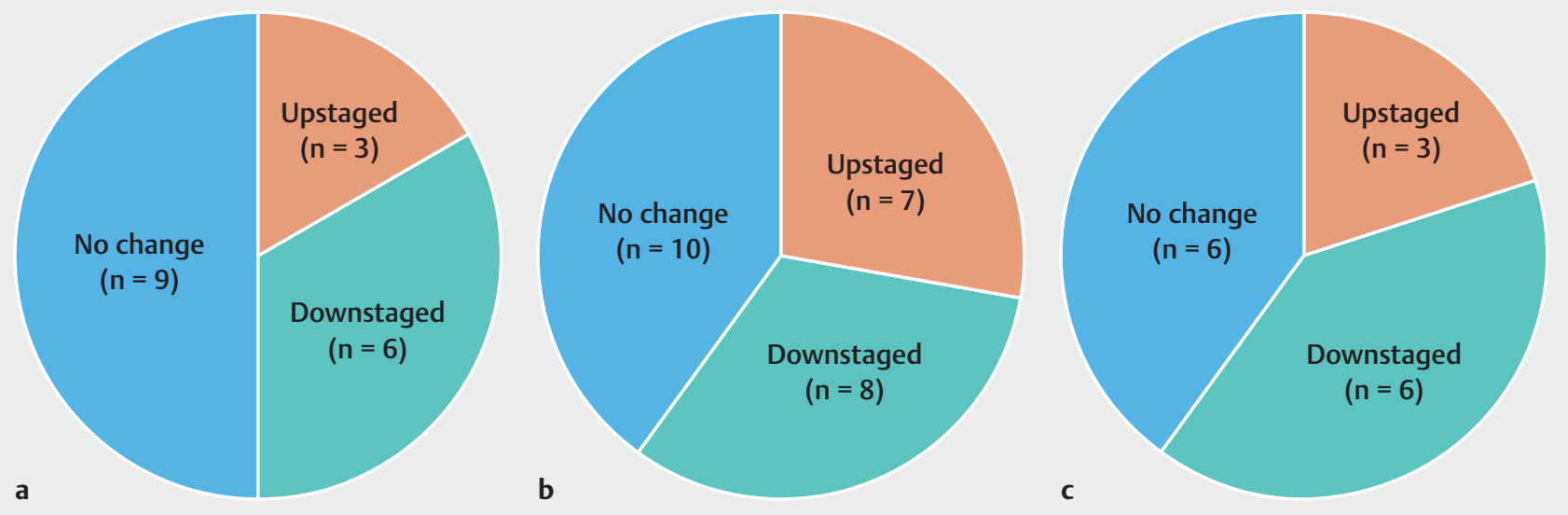

Fig. 3 Impact of TissueCypher results on management decisions in diagnostic subsets of ND, IND, and LGD. Proportions of management plan decisions upstaged, downstaged or not changed after review of TissueCypher results are shown in patients with a non-dysplastic BE; b indefinite for dysplasia, and c low-grade dysplasia. *17 patients had diagnosis of LGD, however, two patients were excluded from this subanalysis due to treatment of higher priority co-morbidity (see Fig. 1 legend).

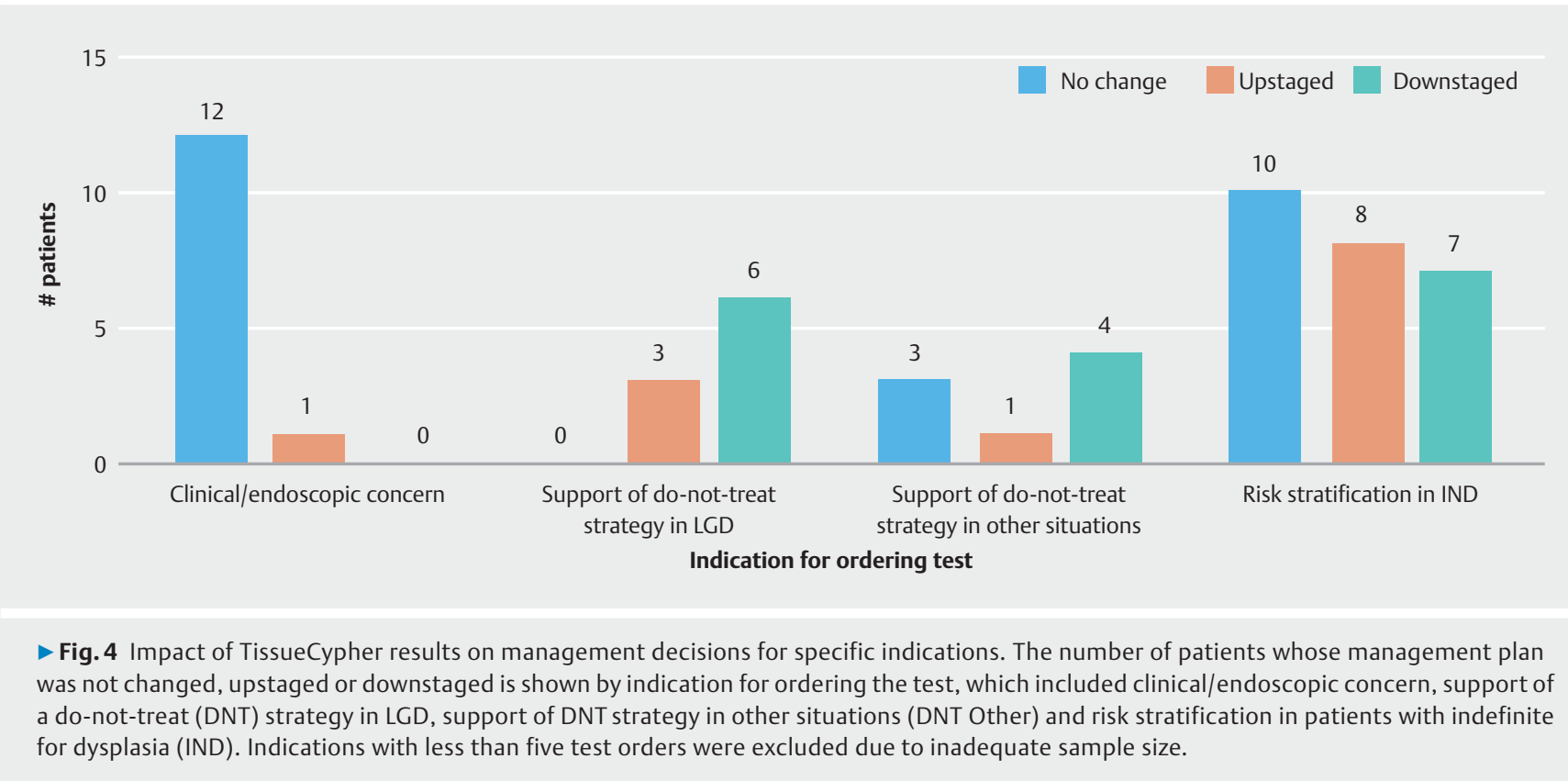

(16.7\% upstaged, $33.3 \%$ downstaged), in IND $60.0 \%$ of decisions were changed (28.0\% upstaged, $32.0 \%$ downstaged), and in patients with LGD $52.9 \%$ decisions were changed $(17.6 \%$ upstaged, $35.3 \%$ downstaged) ( Table 3 and $>$ Fig.3a, -Fig.3b, and $>$ Fig.3c). Downstaging of the management plan after review of test results was more frequent in patients with LGD than with IND and ND. This is likely due to the indication for ordering as the majority of test orders in patients with LGD were to support a do-not-treat strategy. When an intermediate risk result was reported, other clinical factors were taken into account to arrive at a treatment strategy, including comorbidities and history of previous treatment such as refractoriness to endoscopic eradication therapy. This approach led to upstaging of the management plan in three of seven patients who scored TissueCypher intermediate-risk, downstaging in one of seven, and no change in three of seven patients.

A subanalysis was conducted to evaluate the impact of TissueCypher results within specific indications for ordering the test. The impact of test results on management plan was significantly associated with the indication for ordering ( $\mathbf{F i g . 4 ,}$ $P=0.0011)$. The largest impact was observed when the test was ordered to support a do-not-treat (DNT) strategy for LGD (9/11 decisions impacted), DNT in other indications (5/8 deci- 
sions impacted) and for risk stratification in patients with IND (15/25 decisions impacted). Only one of 13 decisions was impacted when the reason for ordering the test was "clinical/ endoscopic concern." The other indication for ordering had sample size of three or less, which was insufficien for this subanalysis.

\section{Discussion}

In this prospective decision impact study, the TissueCypher Barrett's Esophagus Assay significantly influenced decisionmaking in management of patients with BE. Comparison of post-test versus pretest management recommendations in 60 patients with BE showed that the TissueCypher results impacted $55.0 \%$ of management decisions regarding surveillance intervals and therapeutic interventions. The management plan was upstaged after review of TissueCypher results in $21.7 \%$ of patients, which included decisions to intervene with endoscopic eradication therapy (EET) instead of a surveillance only approach. The upstaging of the BE management plan was associated with high-risk results from the TissueCypher test, indicating that the high-risk result may influence physicians to use therapeutic intervention to prevent potential progression to $\mathrm{HGD} / \mathrm{EAC}$, or to increase surveillance in order to monitor these patients more closely. This is a key finding as it suggests that the TissueCypher test has potential clinical utility to improve outcomes by targeting early interventions and close surveillance to an "at-risk" subset of patients, which may have been missed based on the current standard of care The management plan was downstaged after review of TissueCypher results in $33.4 \%$ of patients, and $95 \%$ of these decisions involved changing from EET to surveillance only, with the other $5 \%$ resulting in extension of the surveillance interval from 1 year to 3 years. This is also a critical finding as it indicates that TissueCypher test results have the potential to reduce the overuse of both EET and surveillance endoscopies, which may improve the efficiency of healthcare use in the management of BE.

TissueCypher results impacted management decisions at similar rates in the diagnostic subgroups of non-dysplastic BE (ND), indefinite for dysplasia (IND) and low-grade dysplasia (LGD), suggesting that the test results may be used to guide management decisions in all three of these clinical indications. However, the type of impact differed between the diagnostic subgroups with downstaging occurring more frequently in LGD than in IND and ND BE. This is likely due to the indication for ordering in LGD, which was mostly for support of a do-nottreat strategy.

The finding that high-risk results from TissueCypher led to upstaging of BE management and low-risk results led to downstaging suggests that TissueCypher has the potential to shift the current clinical practice paradigm from surveillance and treatment based on the subjective histopathologic finding of dysplasia to objective risk assessment, with early interventions targeted to high-risk patients, and reduced interventions and less frequent surveillance in low-risk patients. This paradigm shift could improve patient outcomes by preventing EAC in high-risk patients while reducing expenditures on unnecessary procedures and endoscopic surveillance in low-risk patients. A recent cost-effectiveness study compared TissueCypher-guided care versus the current standard of care from the perspective of a large US health care system [27]. Intervention with EET in patients with high-risk TissueCypher scores, in parallel with extension of surveillance intervals to 5 years in patients with low-risk scores, was predicted to be cost-effective within 5 years, while reducing the progression to HGD, EAC and EACrelated deaths by $51.7 \%, 47.1 \%$, and $37.6 \%$, respectively, and also reducing use of endoscopy with biopsy and pathology review by $16.6 \%$.

Overdiagnosis of dysplasia and the resulting interventions and intensive surveillance is costly to the healthcare system. The excess cost associated with overdiagnosis of LGD ranges from $\$ 3,115$ to $\$ 8,072$ per patient in the United States [28], and while EET procedures are considered to be both effective and safe, adverse events occur at a rate of $8.8 \%$ [8]. TissueCypher could be used to target EET and intensive surveillance for at-risk patients, while avoiding overtreatment and overuse of surveillance for LGD and IND. While patients with ND BE are rarely treated with EET, overuse of endoscopic surveillance is common [19]. TissueCypher could be used for ND BE to both reduce the overuse of endoscopic surveillance in low-risk patients and identify the small subset of high-risk patients requiring closer surveillance or EET.

Because the impact of other risk prediction assays on management decisions in $\mathrm{BE}$ has not been reported, there is no other test to which we can directly compare our decision results. However, the impact of precision medicine tests has been extensively studied in other disease areas showing comparable results in terms of how objective risk stratification data can influence decisions made by physicians to manage complex diseases. For example, a systematic review and metaanalysis of 8 studies evaluating the impact of the Oncotype Dx Breast Cancer Recurrence Score found that the test results changed the treatment recommendation regarding adjuvant chemotherapy in $33.4 \%$ of patients with early stage breast cancer [29]. In non-small-cell lung cancer, the VeriStrat test has been shown to lead to a $28.2 \%$ change in treatment recommendations by physicians, leading to a reduction in use of costly and ineffective treatments [30].

The main strengths of this study are the prospective design, and inclusion of ND, IND, and LGD cases for which risk stratification is clinically needed. An additional strength is the study setting, which included a gastrointestinal pathology subspecialty group for interpretation of the biopsy results.

The study limitations are that this was a single-center experience with only two physicians providing management plans before and after receipt of test results. Data on adherence to management plan recommendations or outcomes were not collected. Our center is an expert BE referral center, and the test was ordered selectively rather than consecutively, which resulted in a higher proportion of patients with IND and LGD than would be seen in a typical community practice setting. Additional studies are needed to determine the impact of test results in the community setting where the majority of patients have ND BE. Six of the 60 patients had previously undergone EET and had biop- 
sies showing residual BE when TissueCypher was ordered. While a small study on TissueCypher has been conducted in this setting [21], the majority of clinical studies on the test have been completed in patients who have not been previously treated. Because this was a prospective study assessing physician decision-making, there is a risk of the Hawthorne effect where participants may alter their behavior because they are being observed.

\section{Conclusion}

In summary, objective risk stratification provided by the TissueCypher test had a significant impact on physician decisionmaking in management of patients with BE. Physicians upstaged management of their patients when the test reported high-risk scores and downstaged management recommendations in response to low-risk scores, indicating that the TissueCypher test has clinical utility with the potential to both improve patient outcomes and reduce overuse of procedures in management of BE.

\section{Acknowledgments}

The authors thank Alicia Angelo (Cernostics) for technical assistance in collecting the survey data.

\section{Competing interests}

Dr. Diehl is a consultant for Cernostics and has ownership interest (stock options) in Cernostics, Inc. Dr. Critchley-Thorne has ownership interest (stock, stock options and patents) in Cernostics, Inc.

\section{References}

[1] Thrift AP. Barrett's Esophagus and Esophageal Adenocarcinoma: How Common Are They Really? Dig Dis Sci 2018; 63: 1988-1996

[2] Ferlay J, Colombet M, Soerjomataram I et al. Estimating the global cancer incidence and mortality in 2018: GLOBOCAN sources and methods. Int J Cancer 2018; 144: 1941-1953

[3] Spechler SJ, Sharma P, Souza RF et al. American Gastroenterological Association medical position statement on the management of Barrett's esophagus. Gastroenterology 2011; 140: 1084-1091

[4] Shaheen NJ, Falk GW, Iyer PG et al. ACG Clinical Guideline: Diagnosis and Management of Barrett's Esophagus. Am J Gastroenterol 2015; 111: $30-50$

[5] Qumseya B, Sultan S, Bain P et al. ASGE guideline on screening and surveillance of Barrett's esophagus. Gastrointest Endosc 2019; 90: 335-359 e332

[6] Montgomery E, Bronner MP, Goldblum JR et al. Reproducibility of the diagnosis of dysplasia in Barrett esophagus: a reaffirmation. Hum Pathol 2001; 32: 368-378

[7] Vennalaganti P, Kanakadandi V, Goldblum JR et al. Discordance among pathologists in the United States and Europe in diagnosis of low-grade dysplasia for patients with Barrett's esophagus. Gastroenterology 2017; 152: 564-570 e564

[8] Qumseya BJ, Wani S, Desai M et al. Adverse events after radiofrequency ablation in patients with Barrett's esophagus: a systematic review and meta-analysis. Clin Gastroenterol Hepatol 2016; 14: 1086-1095 e1086

[9] Goldblum JR. Controversies in the diagnosis of Barrett esophagus and Barrett-related dysplasia: one pathologist's perspective. Arch Pathol Lab Med 2010; 134: 1479-1484

[10] Krishnamoorthi R, Singh S, Ragunathan K et al. Factors associated with progression of Barrett's esophagus: a systematic review and meta-analysis. Clin Gastroenterol Hepatol 2018; 16: 1046-1055 e1048

[11] Davison JM, Shah MB, Deitrick C et al. Low-grade dysplasia diagnosis ratio and progression metrics identify variable Barrett's esophagus risk stratification proficiency in independent pathology practices. Gastrointest Endosc 2018; 88: 807-815 e802

[12] Krishnamoorthi R, Mohan BP, Jayaraj M et al. Risk of progression in Barrett's esophagus indefinite for dysplasia: a systematic review and meta-analysis. Gastrointest Endosc 2020; 91: 3-10 e13

[13] Wani S, Falk G, Hall M et al. Patients with nondysplastic Barrett's esophagus have low risks for developing dysplasia or esophageal adenocarcinoma. Clin Gastroenterol Hepatol 2011; 9: 220-227

[14] Desai TK, Krishnan K, Samala $N$ et al. The incidence of oesophageal adenocarcinoma in non-dysplastic Barrett's oesophagus: a meta-analysis. Gut 2012; 61: 970-976

[15] Anaparthy R, Gaddam S, Kanakadandi V et al. Association between length of Barrett's esophagus and risk of high-grade dysplasia or adenocarcinoma in patients without dysplasia. Clin Gastroenterol Hepatol11: 1430-1436

[16] Kambhampati S, Tieu AH, Luber B et al. Risk factors for grogression of Barrett's esophagus to high grade dysplasia and esophageal adenocarcinoma. Sci Rep 2020; 10: 4899

[17] Thrift AP, Kunzmann AT. Time to Tailor surveillance intervals of nondysplastic Barrett's esophagus according to segment length and persistence over multiple endoscopies. Clin Gastroenterol Hepatol 2018; 17: 832-834

[18] Shaheen NJ, Green B, Medapalli RK et al. The perception of cancer risk in patients with prevalent Barrett's esophagus enrolled in an endoscopic surveillance program. Gastroenterology 2005; 129: 429-436

[19] Wani S, Williams JL, Komanduri S et al. Over-utilization of repeat upper endoscopy in patients with non-dysplastic Barrett's esophagus: a quality registry study. Am J Gastroenterol 2019; 144: 1256-1264

[20] Critchley-Thorne RJ, Duits LC, Prichard JW et al. A Tissue Systems Pathology Assay for High-Risk Barrett's Esophagus. Cancer Epidemiol Biomarkers Prev 2016; 25: 958-968

[21] Critchley-Thorne RJ, Davison JM, Prichard JW et al. A tissue systems pathology test detects abnormalities associated with prevalent highgrade dysplasia and esophageal cancer in Barrett's esophagus. Cancer Epidemiol Biomarkers Prev 2017; 26: 240-248

[22] Davison JM, Goldblum J, Grewal US et al. Independent blinded validation of a tissue systems pathology test to predict progression in patients with Barrett's esophagus. Am J Gastroenterol 2020; 115: 843852

[23] Frei NF, Konte K, Bossart EA et al. Independent Validation of tissuecypher to predict future progression in non-dysplastic Barrett's esophagus: a spatial-temporal analysis. Clin Transl Gastroenterol 2020; 11: e00244

[24] Frei NF, Khoshiwal AM, Konte $K$ et al. A Tissue systems pathology test objectively risk stratifies Barrett's esophagus patients with low-grade dysplasia. Am J Gastroenterol 2020: doi:10.14309/ajg.000000000 0001037

[25] Prichard JW, Davison JM, Campbell BB et al. TissueCypher: A systems biology approach to anatomic pathology. J Pathol Informat 2015; 6: 48

[26] DeWard A, Critchley-Thorne RJ. Systems Biology approaches in cancer pathology. Methods Mol Biol 2018; 1711: 261-273 
[27] Hao J, Critchley-Thorne RJ, Diehl DL et al. A cost-effectiveness analysis of an adenocarcinoma risk prediction multi-biomarker assay for patients with Barrett's esophagus. ClinicoEcon Outcomes Res 2019; 11: 623-635

[28] Lash RH, Deas TM Jr, Wians FH Jr. Healthcare cost of over-diagnosis of low-grade dysplasia in Barrett's esophagus. Adv Ther 2016; 33: 684697
[29] Carlson J], Roth JA. The impact of the Oncotype Dx breast cancer assay in clinical practice: a systematic review and meta-analysis. Breast Cancer Res Treat 2013; 141: 13-22

[30] Akerley WL, Arnaud AM, Reddy B et al. Impact of a multivariate serum-based proteomic test on physician treatment recommendations for advanced non-small-cell lung cancer. Curr Med Res Opin 2017; 33: 1091-1097 